\title{
Diagnostic Colposcopy for HPV/DNA Screen Positive Women at the Colposcopy Clinic Family Health Bureau, Colombo
}

\author{
R. Silva ${ }^{1}$, R. Punchihewa ${ }^{2}$, K. C. M. Perera ${ }^{3, *}$, S. A. D. J. Kanchana ${ }^{1}$, L. R. B. Pathirana ${ }^{1}$ \\ ${ }^{1}$ Family Health Bureau, Sri Lanka. \\ ${ }^{2}$ National Hospital for Respiratory Diseases, Welisara, Sri Lanka. \\ ${ }^{3}$ Epidemiology Unit, Ministry of Health, Sri Lanka.
}

How to cite this paper: R. Silva, R. Punchihewa, K. C. M. Perera, S. A. D. J. Kanchana, L. R. B. Pathirana. (2021) Diagnostic Colposcopy for HPV/DNA Screen Positive Women at the Colposcopy Clinic Family Health Bureau, Colombo. International Journal of Clinical and Experimental Medicine Research, 5(2), 232-235.

DOI: 10.26855/ijcemr.2021.04.019

Received: March 25, 2021

Accepted: April 20, 2021

Published: April 30, 2021

*Corresponding author: K. C. M. Perera, Epidemiology Unit, Ministry of Health, Sri Lanka.

Email:

chithranganieperera@yahoo.com

\begin{abstract}
Background: HPV/DNA screening can be used as a primary cervical cancer screening method. The objective of the study was to describe colposcopy results of HPV/DNA screen positive women according to the Asia-Oceania guidelines of follow-up. Method: Descriptive longitudinal study was conducted from 1st of September 2018 to 28th of February 2020 from women, who were undergone for HPV/DNA screening under the pilot project carried out in public health divisions called Medical officer of Health (MOH) areas in Kalutara district. Study population comprised of 35 and 45 year age cohort ever married women in $\mathrm{MOH}$ areas of Kalutara district. HPV/DNA screen positive women with cytology results $\geq$ Atypical Squamous Cells of Undete rmined Significance $(\ell$ ASCUS) were referred to colposcopy. T otal number of 106 women were referred to FHB colposcopy clinic. Total number of 95 women were subjected to biopsy and biopsy screening was carried out by a machine by the Consultant Histopathologist. Results: Percentage of biopsy tissue abnormality among overall HPV/DNA screen positive women with cytology $\geq$ ASCUS was $81.05 \%$ (77/95). Percentage of biopsy tissue abnormality among HPV/DNA genotypes 16 and 18 screen positive women with cytology $\geq$ ASCUS was $92.7 \%(51 / 55)$. Percentage of women subjected to biopsy following abnormal colposcopy results was 89.6\% (95/106). Among all biopsy screened 4.6\% (5/95) were cervical cancer, 8\% (8/95) were High Grade Squamous Intraepithelial Lesions (HGSIL) and 67.36\% were Low Grade Squamous Intraepithelial Lesions (LGSIL) (64/95). Conclusions: Colposcopy and biopsy of HPV/DNA screen positive follow-up has revealed a very high detection rate of Cervical Intraepithelial Neoplasia (CIN). Therefore, HPV/DNA screening as a primary cervical cancer screening method should be considered as it is suitability was well assessed at Sri-Lankan setting.
\end{abstract}

\section{Keywords}

Cervical Cancer Screening, Positive Follow-Up, Diagnostic Test, Colposcopy

\section{Background}

Cervical cancer is the 2nd leading cause of female cancer in Sri Lanka [1]. Hence in 1998, Sri Lanka took an initiative to include screening for cervical cancer with conventional papaniculaou (pap) smear in the Well Woman 
Clinics (WWCs). However, even after 20 years of cervical cancer screening (with pap smears), there is no marked reduction in incidence, morbidity and mortality of cervical cancer in Sri Lanka. Two major drawbacks of the present programme are, the suboptimal sensitivity of the pap smear (53\%) [2] to detect Cervical Intraepithelial Neoplasia (CIN) and the low coverage (53.6\%) [3] of the cervical cancer screening programme.

All cervical cancers are associated with Human papillomavirus (HPV) infection and HPV genotypes 16 and 18 contributes for $70 \%$ of all cervical cancers. Other high risk carcinogenic genotypes of HPV infection are genotypes 31, 33, 35, 39, 45, 51, 52, 56, 58, 59, 66 and 68 (12 pooled high risk). Human papillomavirus/DNA (HPV/DNA) screening test (sensitivity $92.9 \%$ ) can be used as a primary cervical cancer screening method and screen positive women are followed-up according to the Asia-Oceania guidelines [4]. In HPV/DNA screening, screen positive women with cytology result $\geq$ Atypical Squamous Cells of Undetermined Significance (ASCUS) women only are referred for colposcopy, therefore the burden of colposcopy is reduced for $40 \%-50 \%$ in a country.

Asia Oceania Guidelines can be followed [4] for further investigation of the high risk HPV positive women. As per this guideline the HPV/DNA positive women should have pap smear cytology (Figure 1). If cytology is positive for $\geq$ ASCUS, then women should be referred for colposcopy. The HPV/DNA positive cytology negative women should have HPV/DNA test repeated after 1 year. If the test is negative then woman goes back to routine screening. Women with persistent HPV infection have a very high risk of developing CIN2+ lesions [5]. They need to have colposcopy. Even if the colposcopy is normal, women with persistent HPV infection should be followed up yearly with cytology till three consecutive tests are negative [4].

Such triaging approach has the following advantages such as the highly sensitive and objective HPV/DNA test will pick up the true disease more efficiently, due to high negative predictive value of the HPV/DNA test, the HPV/DNA negative women can safely be advised to repeat screening after 10 years or no further screening, if they are negative at 45 years of age, only the HPV/DNA positive women will require cytology, since cytology will be done on the high risk cases, the positive predictive value and the sensitivity of cytology will improve, therefore the cyto-screeners will be less burdened. The objective of the study was to describe colposcopy results of HPV/DNA screen positive women according to the Asia-Oceania guidelines of follow-up [4].

\section{Methods}

Descriptive longitudinal study was conducted from 1st of September 2018 to 28th of February 2020 from 35 and 45 years old women, who were undergone for the HPV/DNA screening under the pilot project carried out in the public health divisions called Medical officer of Health $(\mathrm{MOH})$ areas in Kalutara district.

HPV/DNA screen positive women with cytology results $\geq$ ASCUS were referred to colposcopy clinics at Kethumathie Maternity Hospital, Panadura and Family Health Bureau (FHB), Colombo. Total number of 106 women were referred for FHB colposcopy clinic.

Colposcopy was carried out by using digital colposcope with $1 \%$ acetic acid or $5 \%$ Lugol's iodine. Total number of 95 women were subjected to biopsy and biopsy screening was carried at National Hospital for Respiratory Diseases at Welisara by the Consultant Histopathologist by using a biopsy screening machine.

In primary screening, all HPV/DNA screen positives are referred for cytology (ASC-US triage) according to the positive follow-upalgorithm of Asia Oceania guidelines for the implementation of programs for cervical cancer prevention and control [4)]. HPV/DNA screen positive with cytology $\geq$ ASCUS women only are referred for colposcopy procedure, while the HPV/DNA screen positive but cytology negative women are re-screened for HPV/DNA after one year (Figure 1). Data were entered by using SPSS version 20. Descriptive statistics were used to analysis the data.

\section{Results}

Percentage of biopsy tissue abnormality among overall HPV/DNA screen positive women with cytology $\geq$ ASCUS was 81.05\% (77/95). Percentage of biopsy tissue abnormality among HPV/DNA genotypes 16 and 18 screen positive women with cytology $\geq$ ASCUS was $92.7 \%$ (51/55), while the percentage of biopsy tissue abnormality among HPV/DNA genotypes 12 pooled high risk screen positive women with cytology result $\geq$ ASCUS was $65 \%$ (26/40). Percentage of women subjected to biopsy following abnormal colposcopy results was 89.6\% (95/106). Among all biopsy screened 4.6\% (5/95) were cervical cancer, while 8\% (8/95) were High Grade Squamous Intraepithelial Lesion (HGSIL) and 67.36\% were Low grade Squamous Intraepithelial lesion (LGSIL) (64/95). 


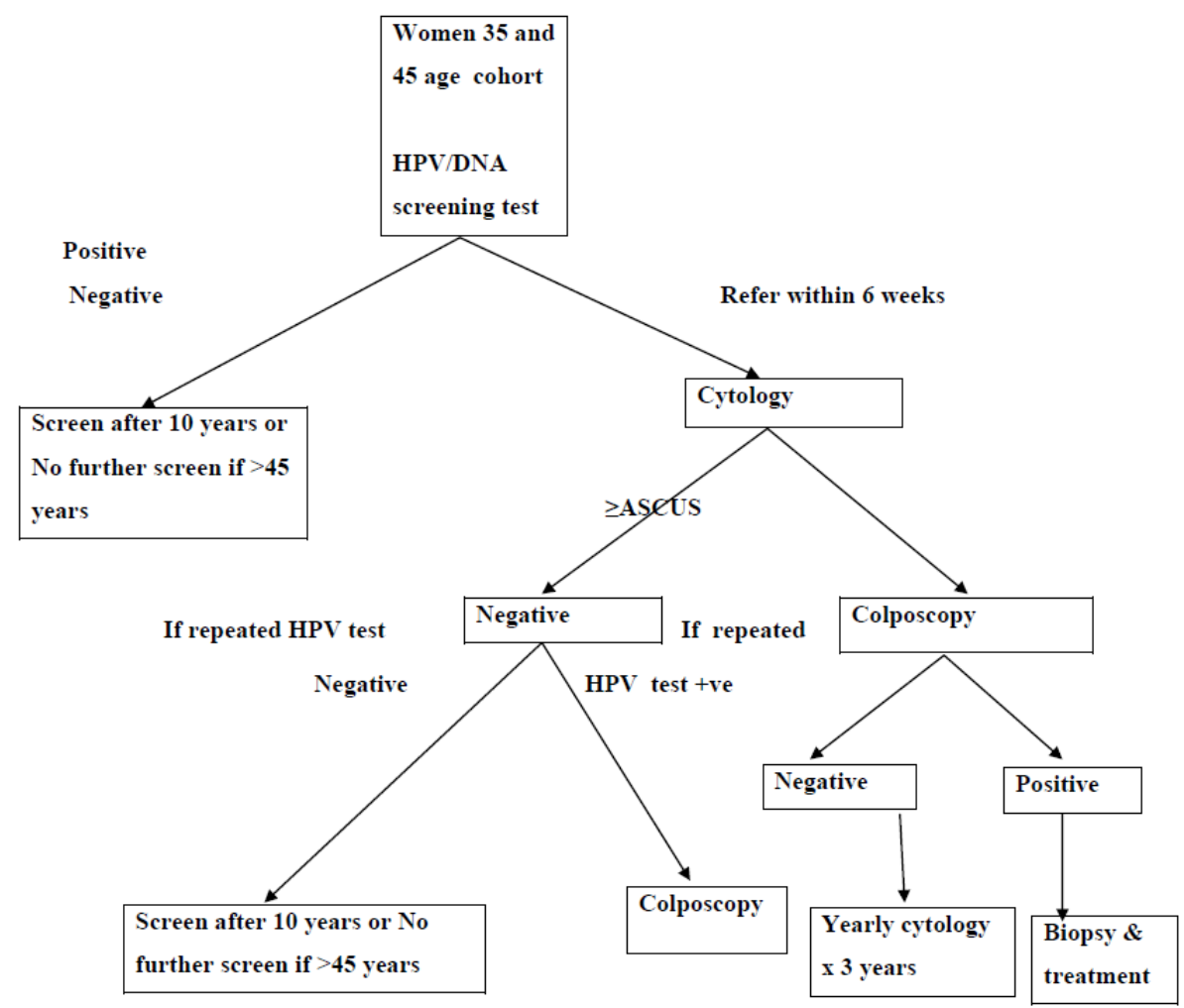

Figure 1. Algorithm of Asia Oceania Guidelines for the Implementation of Programs for Cervical Cancer Prevention and Control for HPV/DNA Screen Positive Follow-up by Hextan et al. (2011).

\section{Conclusions and Recommendations}

HPV/DNA screen positive women with cytology $\geq$ ASCUS were given very high detection of Cervical Intraepithelial Neoplasia (CIN) by colposcopy and biopsy as the sensitivity of the HPV/DNA test was very high. Therefore, HPV/DNA screening as a primary cervical cancer screening method can be considered as it is suitability was well assessed at Sri Lankan setting.

\section{Abbreviations}

ASCUS: Atypical Squamous Cells of Undetermined Significance

CIN: Cervical Intraepithelial Neoplasia

FHB: Family Health Bureau

HGSIL: High Grade Squamous Intraepithelial Lesion

LGSIL: Low Grade Squamous Intraepithelial Lesion

MOH: Medical Officer of Health

\section{Declaration}

\section{1) Acknowledgement}

We are grateful to the study participants and the staff of the Family Health Bureau, colposcopy clinic and the staff of the National Hospital of Respiratory Diseases, laboratory.

\section{2) Funding}

We hereby declare that the cost for specimen collection instruments and reagents (test kits) were funded by the Family Health Bureau, Colombo, Sri-Lanka. There was no any influence from the above mentioned institute during the process of conducting the study or report writing.

\section{3) Availability of data and materials}

The datasets used to analyze in this study is available at corresponding author on reasonable request. 


\section{4) Authors' contribution}

KCMP was participated in the design of the study, coordinated data collection performed the statistical analysis and drafted the version of the manuscript. RS and RP were participated in the design of the study. KCMP was performed the statistical analysis and interpreted data. Both RS and RP were helped to draft the manuscript. All five authors read and approved the final manuscript.

\section{5) Ethical approval and consent to participate}

Ethical clearance was obtained from the Ethics Review Committee (ERC), Faculty of Medicine, University of Colombo, Sri Lanka (ref number EC-17-138). Informed written consent was obtained from each of the selected participants at the field during the initial study. Confidentiality was highly maintained, while handing over individual colposcopy and biopsy result reports. Administrative clearance was obtained from the Director, Family Health Bureau, Colombo and Director, National Hospital for Respiratory Diseases, Welisara.

\section{6) Consent for publication}

Not applicable.

\section{7) Competing interests}

Authors declared that they have no competing interests.

\section{References}

[1] World Health Organization. (2017). Human papillomavirus and related disease report: Sri Lanka. Geneva, Switzerland: World Health Organization; 2017.

[2] World Health Organization. (2015). Strategic framework for the comprehensive control of cancer cervix in South-East Asia Region. Geneva, Switzerland: World Health Organization; 2015.

[3] Family Health Bureau. (2017). Annual report on family health. Colombo, Sri Lanka: Family Health Bureau; 2017.

[4] Hextan, Y. S. N., Garland, S. M., Bhatta, N., Pagliusi, S. R. (2011). Asia Oceania Guidelines for the implementation of programmes for cervical cancer prevention and control. Journal of Cancer Epidemiology, (2011); doi: 10.1115/201/794861.

[5] Bhatla, N., Singla, S., Awasthi, D. (2012). Human papillomavirus deoxyribonucleic acid testing in developed countries. Best Practice \& Research Clinical Obstetrics and Gynaecology, 2012, 26(2): 209-220. 